\title{
Subtelomeric Rearrangements: Presentation of 21 Probands with Emphasis on Familial Cases
}

\author{
Rearranjos Subteloméricos: Apresentação de \\ 21 Probandos, com Ênfase nos Casos Familiares
}

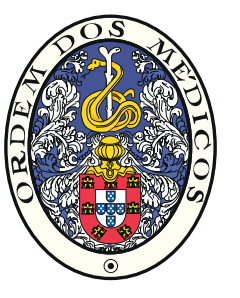

\author{
Ana Rita SOARES $\triangle^{1}$, Gabriela SOARES ${ }^{1}$, Manuela MOTA-FREITAS ${ }^{2,3}$, Natália OLIVA-TELES ${ }^{2,3}$, \\ Ana Maria FORTUNA ${ }^{1,2}$ \\ Acta Med Port 2019 Jul-Aug;32(7-8):529-535 - https://doi.org/10.20344/amp.11466
}

\section{ABSTRACT}

Introduction: Intellectual disability affects $2 \%-3 \%$ of the general population, with a chromosomal abnormality being found in $4 \%-28 \%$ of these patients and a cryptic subtelomeric abnormality in $3 \%-16 \%$. In most cases, these subtelomeric rearrangements are submicroscopic, requiring techniques other than conventional karyotype for detection. They may be de novo or inherited from an affected parent or from a healthy carrier of a balanced chromosomal abnormality. The aim of this study was to characterize patients from our medical genetics center, in whom both a deletion and duplication in subtelomeric regions were found.

Material and Methods: Clinical and cytogenetic characterization of 21 probands followed at our center, from 1998 until 2017, with subtelomeric rearrangements.

Results: There were 21 probands from 19 families presenting with intellectual disability and facial dysmorphisms. Seven had behavior changes, five had epilepsy and 14 presented with some other sign or symptom. Four had chromosomal abnormalities detected by conventional karyotype and four were diagnosed by array-comparative genomic hybridization. In four cases, parental studies were not possible. The online mendelian inheritance in man classification was provided whenever any of the phenotypes (deletion or duplication syndrome) was dominant.

Discussion: Patients and relevant family members were clinically and cytogenetically characterized. Although rare, subtelomeric changes are a substantial cause of syndromic intellectual disability with important familial repercussions. It is essential to remember that a normal array-comparative genomic hybridization result does not exclude a balanced rearrangement in the parents.

Conclusion: Parental genetic studies are essential not only for a complete characterization of the rearrangement, but also for accurate genetic counselling and screening of family members at risk for recurrence.

Keywords: Intellectual Disability/genetics; Subtelomeric Rearrangements Gene Rearrangement/genetics; Telomere/genetics

\section{RESUMO}

Introdução: O défice intelectual afeta $2 \%-3 \%$ da população geral, sendo encontrada uma alteração cromossómica em $4 \%-28 \%$ dos casos e uma alteração subtelomérica em 3\% - 16\%. Estas alterações subteloméricas são, na maioria dos casos, submicroscópicas, não sendo detetadas no cariótipo convencional. Podem ser de novo ou herdadas de um progenitor afetado ou de um progenitor saudável portador de um rearranjo equilibrado. O objetivo deste estudo foi caracterizar os doentes seguidos no nosso centro de genética médica com uma deleção e uma duplicação nas regiões subteloméricas.

Material e Métodos: Caracterização clínica e citogenética de 21 probandos com alterações subteloméricas seguidos no nosso centro entre 1998 e 2017.

Resultados: Foram caracterizados 21 probandos que apresentavam défice intelectual e dismorfia facial, pertencentes a 19 famílias. Sete tinham alteraç̃̃es do comportamento, cinco epilepsia e 14 outro sinal ou sintoma. Quatro tinham alterações no cariótipo e quatro foram diagnosticados por array-comparative genomic hybridization. Em quatro famílias não foi possível o estudo dos progenitores. Quando um dos fenótipos era dominante (síndrome de deleção ou duplicação), foi atribuída a classificação online mendelian inheritance in man.

Discussão: Foi realizada classificação dos doentes e das famílias. As alterações nas regiões subteloméricas são, apesar de raras, uma causa substancial para défice intelectual sindrómico com repercussões familiares importantes. É essencial lembrar que um arraycomparative genomic hybridization normal não exclui um rearranjo equilibrado familiar.

Conclusão: O estudo dos progenitores é essencial não só para caracterização completa do rearranjo mas também para um aconseIhamento genético preciso e identificação de familiares em risco de recorrência.

Palavras-chave: Deficiência Intelectual/genética; Rearranjo Génico/genética; Telómero/genética

\section{INTRODUCTION}

Intellectual disability, previously known as mental retardation, affects $2 \%-3 \%$ of the general population..$^{1-3}$ The Diagnostic and Statistical Manual of Mental Disorders (DSM)-5 defines intellectual disability as a disorder with onset during the developmental period that includes both intellectual and adaptive functioning deficits in conceptual, social and practical domains. It can be classified into mild, moderate, severe and profound intellectual disability, according to its severity. ${ }^{4,5}$ The aetiology of ID is heterogeneous

1. Unidade de Genética Médica. Centro de Genética Médica Jacinto Magalhães. Centro Hospitalar Universitário do Porto. Porto. Portugal.

2. Unidade de Citogenética. Centro de Genética Médica Jacinto Magalhães. Centro Hospitalar Universitário do Porto. Porto. Portugal.

3. Unidade Multidisciplinar de Investigação Biomédica. Instituto de Ciências Biomédicas Abel Salazar. Universidade do Porto. Porto. Portugal.

$\triangle$ Autor correspondente: Ana Rita Soares. ana.rita.soares@chporto.min-saude.pt

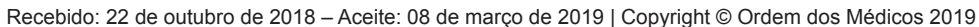


and includes both genetic and environmental causes, ${ }^{2}$ and sometimes it is multifactorial. Most patients, particularly those with moderate/severe ID and with dysmorphic features/congenital abnormalities, have a genetic aetiology, which can be a chromosomal imbalance or a single gene pathogenic variant. A chromosomal abnormality is found in $4 \%-28 \%$ of patients with ID..$^{2,5}$ Subtelomeric regions are gene-rich chromosomal regions with a highly repetitive structure, frequently involved in chromosomal rearrangements. ${ }^{6,7}$ Cryptic subtelomeric abnormalities, syndromic or isolated, are well known causes of ID, and are responsible for $3 \%-16 \%$ of diagnoses.

These subtelomeric rearrangements are predominantly submicroscopic [i.e. smaller than 5 megabases (Mb)], requiring techniques other than conventional karyotype for detection. Karyotype with fluorescent in situ hybridization (FISH) was the first diagnostic approach for these subtelomeric abnormalities. Multiplex ligation-dependent probe amplification (MLPA), a semi-quantitative methodology, allowed a better characterization of duplications or deletions. Currently, array-comparative genomic hybridization $(\mathrm{aCGH})$ is used as the first tier-test for these patients. ${ }^{8,9}$ All the above techniques have been used as routine tests for identifying these patients. . $^{2,3,10}$

An accurate family history may show important clues for a possible inherited rearrangement, mainly more than one family member affected or history of recurrent pregnancy losses. In fact, these subtelomeric abnormalities may be de novo or inherited. An unbalanced rearrangement may be inherited from an affected parent or from a healthy carrier of a balanced chromosomal abnormality. Parental genetic studies are thus essential not only for a complete characterization of the rearrangement (de novo or inherited) but also, and more importantly, for accurate genetic counselling and screening of family members at risk for recurrence.

Following the identification of a patient (case 1) in whom aCGH showed the presence of a deletion and a duplication in subtelomeric regions, and complementary studies in parents confirmed that the mother was a carrier of a balanced subtelomeric cryptic translocation, we decided to review and characterize the patients from our medical genetics center, from clinical and cytogenetic perspectives, in whom both deletion and duplication on subtelomeric regions were found. With this work, our main objective was to raise awareness for these rare genetic syndromes as well as to the importance of familial studies.

\section{MATERIAL AND METHODS}

Our study included 21 probands followed at our medical genetics center, from 1998 until 2017, who have simultaneously a deletion and a duplication in chromosomal subtelomeric regions. We describe gender, age at first consultation, somatometry, craniofacial and other dysmorphisms or congenital abnormalities, family history, as well as cytogenetic findings in patients and parents.

Written informed consent was obtained for all published photos.

\section{RESULTS}

Results are summarized in Table 1, Table 2 and Figs. 1 to 3 . Twenty one probands (eight males and 13 females) from 19 families presented with developmental delay/intellectual disability (11 cases with severe, four with moderate and six with mild ID) and facial dysmorphisms (Table $1 \mathrm{~A}$ and Fig. 4). Seven had behaviour changes, five had epilepsy and 14 presented with some other sign or symptom. Cases 4 to 6 represent a brother, a sister and a maternal cousin from the same family. Four cases presented with visible G-bands by trypsin using Leishman (GTL) banding chromosomal abnormalities: three of them with subtelomeric abnormalities [case 18: 46,XY,der(6)t(6;9) (q27;p12); case 19: 46,XX,der(8)t(4;8)(p16,1;p23,1); case 21: 46,XX,der(3)t(3;11)(p26.2;p15.4)pat], and case 15 had a balanced and non-pathogenic robertsonian translocation [45,XX,rob(13;14)(q10;q10)], in addition to the subtelomeric submicroscopic abnormalities. Four cases were diagnosed by aCGH and the others by MLPA/FISH. In four cases, the parental clinical and cytogenetic studies were not possible. Of the cases in which family studies were possible, six were de novo events, seven were inherited from a balanced cryptic rearrangement and three probands inherited the abnormality from a similarly affected parent. In case 21 the translocation was shown to have been inherited from the paternal grandfather. Most cases presented with phenotypic characteristics of both deletion and duplication syndromes involved in the rearrangement. Therefore, the OMIM classification was provided whenever any of the phenotypes was dominant (Tables 1A and 1B).

\section{DISCUSSION}

Subtelomeric regions are gene-rich chromosomal regions responsible for a significant number of patients with syndromic ID. At Centro Hospitalar Universitário do Porto, 21 cases with a subtelomeric chromosomal rearrangement have been diagnosed and characterized, and accurate genetic counselling was offered.

Case 1 presented with a phenotype more compatible with chromosome 16p13.3 duplication syndrome. This

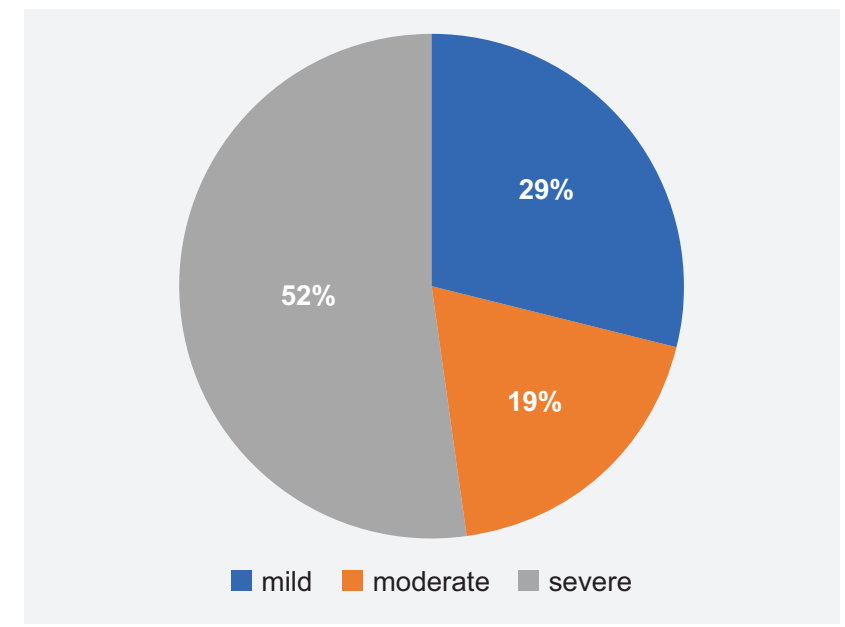

Figure 1 - Intellectual disability 
Table 1A - Clinical and cytogenetic characterization of our patients' cohort

\begin{tabular}{|c|c|c|c|c|c|c|c|}
\hline Case & Age $^{*}$ & Sex & Facial Dysmorphisms & DD/ID & $\begin{array}{l}\text { Behaviour } \\
\text { changes }\end{array}$ & Epilepsy & Other \\
\hline 1 & $13 m$ & $\mathrm{~F}$ & $\begin{array}{l}\text { High forehead, small, upslanting palpebral fissures, } \\
\text { flat philtrum, small mouth with thin lips }\end{array}$ & mild & - & - & Hypotonia \\
\hline 2 & $25 y$ & $\mathrm{~F}$ & $\begin{array}{l}\text { Frontal bossing, hypertelorism, long nose with } \\
\text { overhanging nasal tip }\end{array}$ & severe & - & + & - \\
\hline 3 & $29 y$ & M & Round face, synophrys, large, smooth philtrum & severe & + & + & Short stature \\
\hline 4 & $12 \mathrm{~m}$ & M & $\begin{array}{l}\text { Mild plagiocephaly and facial asymmetry, flat midface, } \\
\text { straight eyebrows, deeply set eyes with downslanting } \\
\text { palpebral fissures, small nose with flat nasal bridge } \\
\text { and bulbous tip, downturned corners of mouth }\end{array}$ & severe & + & + & - \\
\hline 5 & $11 \mathrm{~m}$ & $\mathrm{~F}$ & $\begin{array}{l}\text { Mild plagiocephaly and facial asymmetry, flat midface, } \\
\text { straight eyebrows, deeply set eyes with downslanting } \\
\text { palpebral fissures, small nose with flat nasal bridge } \\
\text { and bulbous tip, downturned corners of mouth }\end{array}$ & severe & + & + & - \\
\hline 6 & $7 \mathrm{~m}$ & $\mathrm{~F}$ & $\begin{array}{l}\text { Mild plagiocephaly and facial asymmetry, flat midface, } \\
\text { straight eyebrows, deeply set eyes with downslanting } \\
\text { palpebral fissures, small nose with flat nasal bridge } \\
\text { and bulbous tip, downturned corners of mouth }\end{array}$ & severe & + & + & - \\
\hline 7 & $3 y$ & $\mathrm{~F}$ & $\begin{array}{l}\text { Frontal bossing, arched eyebrows, flat nasal bridge, } \\
\text { small low-set dysplastic ears }\end{array}$ & severe & - & - & $\begin{array}{l}\text { Obesity, type } 4 \\
\text { brachydactyly, } \\
\text { hypotonia }\end{array}$ \\
\hline 8 & $10 y$ & $\mathrm{~F}$ & $\begin{array}{l}\text { Frontal bossing, arched eyebrows, flat nasal bridge, } \\
\text { small low-set dysplastic ears }\end{array}$ & severe & + & - & $\begin{array}{l}\text { Short stature, type } 4 \\
\text { brachydactyly }\end{array}$ \\
\hline 9 & $39 y$ & $\mathrm{~F}$ & $\begin{array}{l}\text { Downslanting palpebral fissures, arched eyebrows, } \\
\text { thick lips }\end{array}$ & severe & - & - & Overweight \\
\hline 10 & $12 y$ & $\mathrm{~F}$ & Small eyes, long nose, large teeth & severe & + & - & Severe scoliosis \\
\hline 11 & $22 m$ & M & $\begin{array}{l}\text { High forehead, small low-set ears, thick eyebrows, } \\
\text { small nose, micrognathia }\end{array}$ & severe & - & - & $\begin{array}{l}\text { Cleft palate, } \\
\text { hypotonia, ataxia }\end{array}$ \\
\hline 12 & $3 y$ & M & $\begin{array}{l}\text { Macrocephaly with frontal bossing, low-set ears, } \\
\text { telecanthus, hyperthelorism, decentered elliptical } \\
\text { pupils }\end{array}$ & mild & - & - & $\begin{array}{l}\text { MVP, Rieger's } \\
\text { anomaly }\end{array}$ \\
\hline 13 & $39 y$ & $\mathrm{~F}$ & $\begin{array}{l}\text { Rough face, blepharophimosis, telecanthus, long } \\
\text { nose with overhanging nasal tip, prognathism, large, } \\
\text { protruding tongue }\end{array}$ & severe & - & - & Scoliosis \\
\hline 14 & $8 y$ & M & $\begin{array}{l}\text { Microcephaly, small forehead, prominent nose with } \\
\text { hypoplastic nares, microretrognathia }\end{array}$ & moderate & - & - & - \\
\hline 15 & $11 y$ & $\mathrm{~F}$ & $\begin{array}{l}\text { Microcephaly, bitemporal narrowing, pointed nose, } \\
\text { microretrognathia, mentonian groove }\end{array}$ & moderate & + & - & Failure to thrive \\
\hline 16 & $23 y$ & M & $\begin{array}{l}\text { Small forehead, dysplastic ears, arched eyebrows, } \\
\text { epicanthus, telecanthus, large mouth with microdontia } \\
\text { and some conical teeth, micrognathia }\end{array}$ & mild & - & - & - \\
\hline 17 & $4 y$ & $\mathrm{~F}$ & Blepharophimosis, left ptosis, epicanthus, strabismus & mild & - & - & - \\
\hline 18 & $3 m$ & $M$ & $\begin{array}{l}\text { High forehead, simple cup-shaped ears, epicanthus, } \\
\text { small palpebral fissures, broad nose with bulbous tip, } \\
\text { microretrognathia }\end{array}$ & moderate & - & - & $\begin{array}{l}\text { Failure to thrive, } \\
\text { kyphoscoliosis, } \\
\text { short 5th finger with } \\
\text { clinodactily, hypotonia }\end{array}$ \\
\hline 19 & $3 y$ & $F$ & $\begin{array}{l}\text { Broad forehead, midface hypoplasia, big cheeks, } \\
\text { spoon ear, depressed nasal bridge, strabismus }\end{array}$ & mild & - & - & $\begin{array}{l}\text { Foetal growth } \\
\text { restriction, speech } \\
\text { delay }\end{array}$ \\
\hline 20 & $11 \mathrm{~m}$ & $\mathrm{M}$ & $\begin{array}{l}\text { Brachycephaly, round face, epicanthus, upslanting } \\
\text { palpebral fissures, bulbous nose with anteverted } \\
\text { nares, thin lips, low-set ears }\end{array}$ & mild & - & - & $\begin{array}{l}\text { Short puffy hands } \\
\text { and feet, right } \\
\text { cryptorchidism, } \\
\text { hypotonia, mongolian } \\
\text { spot }\end{array}$ \\
\hline 21 & $1 \mathrm{~m}$ & $\mathrm{~F}$ & $\begin{array}{l}\text { High front, small, low-set and posteriorly rotated } \\
\text { ears, downslanting palpebral fissures, bulbous nose } \\
\text { tip with wide anteverted nares, large mouth with } \\
\text { macroglossia, mild micrognathia }\end{array}$ & moderate & - & - & $\begin{array}{l}\text { Loose skin, umbilical } \\
\text { hernia, strabismus, } \\
\text { scoliosis }\end{array}$ \\
\hline
\end{tabular}

*age at first consultation; ${ }^{* *}$ OMIM dominant phenotoype when it was possible to characterize; y: years-old; m: months-old; M/F: male/ female; DD/ID: developmental delay/intellectual disability; mat/pat: maternal/paternal; dn: de novo; unk: unknown; del/dup: deletion/duplication; (un)bal: (un)balanced; Sdr: syndrome. 
syndrome is characterized by ID, facial dysmorphisms (high forehead, sparse eyebrows, blepharophimosis with palpebral ptosis, short nose, everted upper lip, high-arched palate and cupped ears), pre and postnatal growth deficiency, clef palate, congenital heart defects and urogenital abnormalities. ${ }^{11}$

Case 2 presented mainly with a phenotype consistent with Phelan-McDermid syndrome, characterized by neonatal hypotonia, global developmental delay, moderate to severe ID, absent or severely impaired speech, normal to accelerated growth, large fleshy hands, dysplastic toenails, decreased sudoresis with tendency to overheat and behaviour changes (chewing, decreased perception of pain, autistic-like features). ${ }^{12}$

Case 14 presented with chromosome 22q13 duplication syndrome, the duplication occurring in the same chromosomal region where there was a deletion in case 2 ; this syndrome was described in patients presenting with global developmental delay, prenatal and postnatal growth retardation, hypotonia, facial dysmorphisms (microcephaly,

Table 1B - Prevalence of children from 0 to 9 years old exposed to SHS at home according to parents' tobacco consumption, by region

\begin{tabular}{|c|c|c|c|c|c|c|}
\hline Case & Karyotype & $\begin{array}{l}\text { Chromosomal } \\
\text { abnormalities }\end{array}$ & $\begin{array}{l}\text { Genetic } \\
\text { Study }\end{array}$ & Type & & Dominant phenotype ${ }^{* *}$ \\
\hline 1 & $46, x X$ & $\begin{array}{l}\text { del2q37.3, } \\
\text { dup16p13.3 }\end{array}$ & aCGH & $\begin{array}{l}\text { mat } \\
\text { translocation }\end{array}$ & bal & $\begin{array}{l}\text { \#613458 Chromosome } \\
\text { 16p13.3 Duplication Sdr }\end{array}$ \\
\hline 2 & $46, X X$ & $\begin{array}{l}\text { del22q13.33, } \\
\text { dup19q13.4 }\end{array}$ & $\begin{array}{l}\text { MLPA, } \\
\text { FISH }\end{array}$ & $\begin{array}{l}\text { mat } \\
\text { translocation }\end{array}$ & bal & $\begin{array}{l}\text { \#606232 Phelan- } \\
\text { McDermid Sdr }\end{array}$ \\
\hline 3 & $46, X Y$ & $\begin{array}{l}\text { del5p15.33, } \\
\text { dup18p11.32 }\end{array}$ & $\begin{array}{l}\text { MLPA, } \\
\text { FISH }\end{array}$ & $\begin{array}{l}\text { mat } \\
\text { translocation }\end{array}$ & unbal & $\begin{array}{l}\# 123450 \text { Chromosome } 5 p \\
\text { Deletion Sdr }\end{array}$ \\
\hline 4 & $46, X Y$ & $\begin{array}{l}\text { del1p36.33, } \\
\text { dup14q32.3 }\end{array}$ & $\begin{array}{l}\text { MLPA, } \\
\text { FISH }\end{array}$ & $\begin{array}{l}\text { mat } \\
\text { translocation }\end{array}$ & bal & $\begin{array}{l}\text { \#607872 Chromosome } \\
\text { 1p36 Deletion Sdr }\end{array}$ \\
\hline 5 & $46, x X$ & $\begin{array}{l}\text { del1p36.33, } \\
\text { dup14q32.3 }\end{array}$ & $\begin{array}{l}\text { MLPA, } \\
\text { FISH }\end{array}$ & $\begin{array}{l}\text { mat } \\
\text { translocation }\end{array}$ & bal & $\begin{array}{l}\text { \#607872 Chromosome } \\
\text { 1p36 Deletion Sdr }\end{array}$ \\
\hline 6 & $46, X X$ & $\begin{array}{l}\text { del1p36.33, } \\
\text { dup14q32.3 }\end{array}$ & $\begin{array}{l}\text { MLPA, } \\
\text { FISH }\end{array}$ & $\begin{array}{l}\text { pat } \\
\text { translocation }\end{array}$ & bal & $\begin{array}{l}\text { \#607872 Chromosome } \\
\text { 1p36 Deletion Sdr }\end{array}$ \\
\hline 7 & $46, X X$ & $\begin{array}{l}\text { del2q37.3, } \\
\text { dup12q24.33 }\end{array}$ & $\begin{array}{l}\text { MLPA, } \\
\text { FISH }\end{array}$ & unk & unk & $\begin{array}{l}\text { \#600430 Chromosome } \\
\text { 2q37 Deletion Sdr }\end{array}$ \\
\hline 8 & $46, X X$ & $\begin{array}{l}\text { del2q37.3, } \\
\text { dup17q25.3 }\end{array}$ & $\begin{array}{l}\text { MLPA, } \\
\text { FISH }\end{array}$ & $\begin{array}{l}\text { mat } \\
\text { translocation }\end{array}$ & bal & $\begin{array}{l}\text { \#600430 Chromosome } \\
\text { 2q37 Deletion Sdr }\end{array}$ \\
\hline 9 & $46, X X$ & $\begin{array}{l}\text { del12p13.33, } \\
\text { dup12q24.33 }\end{array}$ & $\begin{array}{l}\text { MLPA, } \\
\text { FISH }\end{array}$ & pat inversion & bal & \\
\hline 10 & $46, X X$ & $\begin{array}{l}\text { del8p23.3, } \\
\text { dup4p16.3 }\end{array}$ & $\begin{array}{l}\text { MLPA, } \\
\text { FISH }\end{array}$ & dn & $\mathrm{dn}$ & \\
\hline 11 & $46, X Y$ & $\begin{array}{l}\text { del6p25.3, } \\
\text { dup17q25.3 }\end{array}$ & $\mathrm{FISH}$ & $\mathrm{dn}$ & $\mathrm{dn}$ & $\begin{array}{l}\text { \#612582 Chromosome } \\
\text { 6pter-p24 Deletion Sdr }\end{array}$ \\
\hline 12 & $46, X Y$ & $\begin{array}{l}\text { del6p25.3, } \\
\text { dupXq28/Yq12 }\end{array}$ & $\begin{array}{l}\text { MLPA, } \\
\text { FISH }\end{array}$ & $\begin{array}{l}\text { pat } \\
\text { translocation }\end{array}$ & unbal & $\begin{array}{l}\text { \#612582 Chromosome } \\
\text { 6pter-p24 Deletion Sdr }\end{array}$ \\
\hline 13 & $46, X X$ & $\begin{array}{l}\text { del18p11.21, } \\
\text { dup11p15.5 }\end{array}$ & $\begin{array}{l}\text { MLPA, } \\
\text { FISH }\end{array}$ & unk & unk & \\
\hline 14 & $46, X Y$ & $\begin{array}{l}\text { del6q27, } \\
\text { dup22q13.33 }\end{array}$ & $\begin{array}{l}\text { MLPA, } \\
\text { FISH }\end{array}$ & unk & unk & $\begin{array}{l}\text { \#615538 Chromosome } \\
22 q 13 \text { Duplication Sdr }\end{array}$ \\
\hline 15 & $45, X X, \operatorname{rob}(13 ; 14)(q 10 ; q 10)$ & $\begin{array}{l}\text { del15q26.3, } \\
\text { dup19p13.3 }\end{array}$ & aCGH & $\begin{array}{l}\text { mat deletion, } \\
\text { pat duplication }\end{array}$ & unbal & $\begin{array}{l}\text { \#612626 Chromosome } \\
\text { 15q26-qter Deletion Sdr }\end{array}$ \\
\hline 16 & $46, X Y$ & $\begin{array}{l}\text { del4q35.2, } \\
\text { dup1p36.33 }\end{array}$ & $\begin{array}{l}\text { MLPA, } \\
\text { FISH }\end{array}$ & unk & unk & \\
\hline 17 & $46, X X$ & $\begin{array}{l}\text { del14q32.33, } \\
\text { dup20q13.33 }\end{array}$ & $\begin{array}{l}\text { MLPA, } \\
\text { FISH }\end{array}$ & $\mathrm{dn}$ & $\mathrm{dn}$ & \\
\hline 18 & $46, \mathrm{XY}, \operatorname{der}(6) \mathrm{t}(6 ; 9)(\mathrm{q} 27 ; \mathrm{p} 12)$ & $\begin{array}{l}\text { del6q27, } \\
\text { dup9p12 }\end{array}$ & $\begin{array}{l}\text { Karyotype, } \\
\text { FISH }\end{array}$ & $\mathrm{dn}$ & $\mathrm{dn}$ & \\
\hline 19 & $46, X X, \operatorname{der}(8)(4 ; 8)(\mathrm{p} 16,1 ; \mathrm{p} 23,1)$ & $\begin{array}{l}\text { del8p23.3p23.1, } \\
\text { dup4p16.3p16.1 }\end{array}$ & $\begin{array}{l}\text { aCGH, } \\
\text { Karyotype }\end{array}$ & $\mathrm{dn}$ & $\mathrm{dn}$ & \\
\hline 20 & $46, X Y$ & $\begin{array}{l}\text { del18q22.3q23, } \\
\text { dup21q22.2q22.3 }\end{array}$ & aCGH & $\mathrm{dn}$ & $\mathrm{dn}$ & \#190685 Down Sdr \\
\hline 21 & $46, X X, \operatorname{der}(3) t(3 ; 11)(p 26.2 ; p 15.4)$ pat & $\begin{array}{l}\text { del3p26.2, } \\
\text { dup11p15.4 }\end{array}$ & $\begin{array}{l}\text { Karyotype, } \\
\text { MLPA }\end{array}$ & $\begin{array}{l}\text { pat } \\
\text { translocation }\end{array}$ & $\begin{array}{l}\text { bal and } \\
\text { pat } \\
\text { inherited }\end{array}$ & $\begin{array}{l}\text { \#130650 Beckwith- } \\
\text { Wiedemann Sdr }\end{array}$ \\
\hline
\end{tabular}

*age at first consultation; ${ }^{* *}$ OMIM dominant phenotoype when it was possible to characterize; y: years-old; m: months-old; M/F: male/ female; DD/ID: developmental delay/intellectual disability; mat/pat: maternal/paternal; dn: de novo; unk: unknown; del/dup: deletion/duplication; (un)bal: (un)balanced; Sdr: syndrome. 
micrognathia, hypertelorism and low-set ears), cleft lip and palate, congenital heart disease, kidney, genital and skeletal abnormalities. However, different sized duplications have shown variable phenotypic severity and inconsistent phenotype even in affected members of the same family. ${ }^{13}$

Case 3 presented with severe developmental delay, behaviour changes, epilepsy, short stature and facial dysmorphisms (round face, micrognathia, epicanthic folds, hypertelorism). This phenotype is mainly caused by the $5 p 15.33$ deletion, compatible with chromosome $5 p$ deletion syndrome. ${ }^{14}$

Cases 4, 5 and 6 are three affected individuals (a boy and two girls) within the same family. They all presented with a phenotype characteristic of chromosome 1p36 deletion syndrome: this is a well known syndrome comprising a characteristic facial appearance (microcephaly, brachycephaly, prominent forehead, midface hypoplasia, deep-set eyes with straight eyebrows, thick ear helices, flat nose and nasal bridge and pointed chin), hypotonia, developmental delay, growth retardation, seizures, hearing impairment, visual problems, cardiovascular and limb abnormalities. ${ }^{15,16}$

Both cases 7 and 8 presented with some phenotypic features of chromosome 2q37 deletion syndrome, which is characterized by facial dysmorphisms (prominent forehead, round face, midface hypoplasia, sparse arched eyebrows,
Table 2 - Demographic data

\begin{tabular}{lccc}
\hline & $<\mathbf{2 y}$ & $\mathbf{2 - 1 8 y}$ & $>18 \mathbf{y}$ \\
\hline $\mathbf{M}$ & 4 & 2 & 2 \\
$\mathbf{F}$ & 4 & 6 & 3 \\
Total & 8 & 8 & 5 \\
\hline
\end{tabular}

Y: years-old; m: months-old; M/F: male/female

deep-set eyes, depressed nasal bridge, thin upper lip and dysmorphic ears), short stature, obesity, brachydactyly type E, mild to moderate ID and behavioural problems. Other major malformations may occur, including congenital heart disease, central nervous system malformations, and gastrointestinal or genitourinary abnormalities. ${ }^{17,18}$

Vaglio et al have described a case of a boy presenting with partial monosomy $12 p$ and trisomy $12 q$ and compared him with other cases described in the literature. As in our case 9, patients with this chromosomal abnormality show clinical features that are characteristic of both deletion and duplication syndromes. It was not possible to detect predominance of either. All of these cases occurred as a consequence of a balanced inversion present in a healthy parent. ${ }^{19}$

The chromosomal study of cases 10 and 19 revealed in both cases a deletion in 8p23.3 and a duplication in $4 p 16.3$.

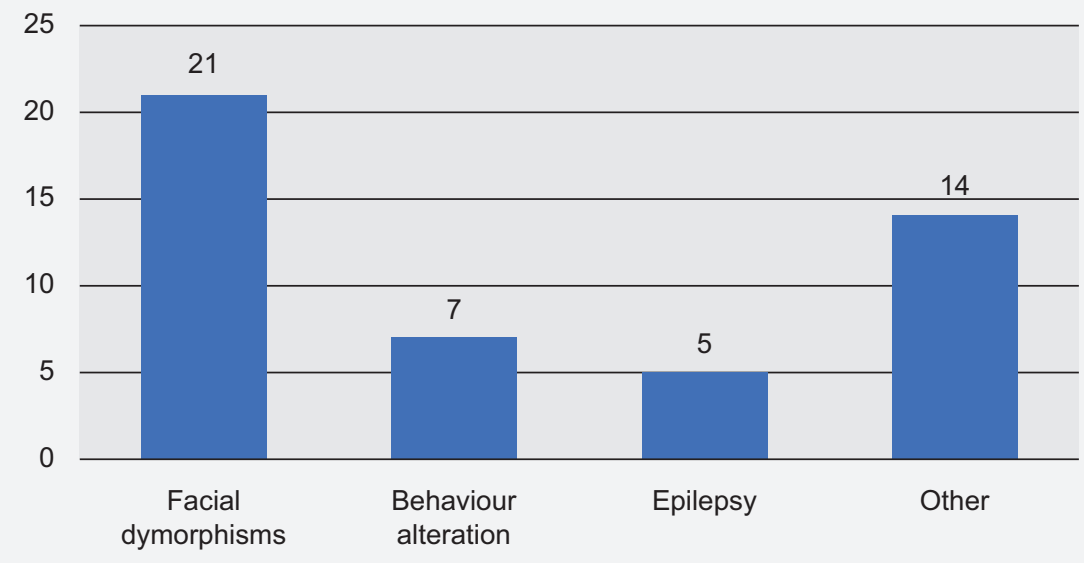

Figure 2 - Clinical characteristics

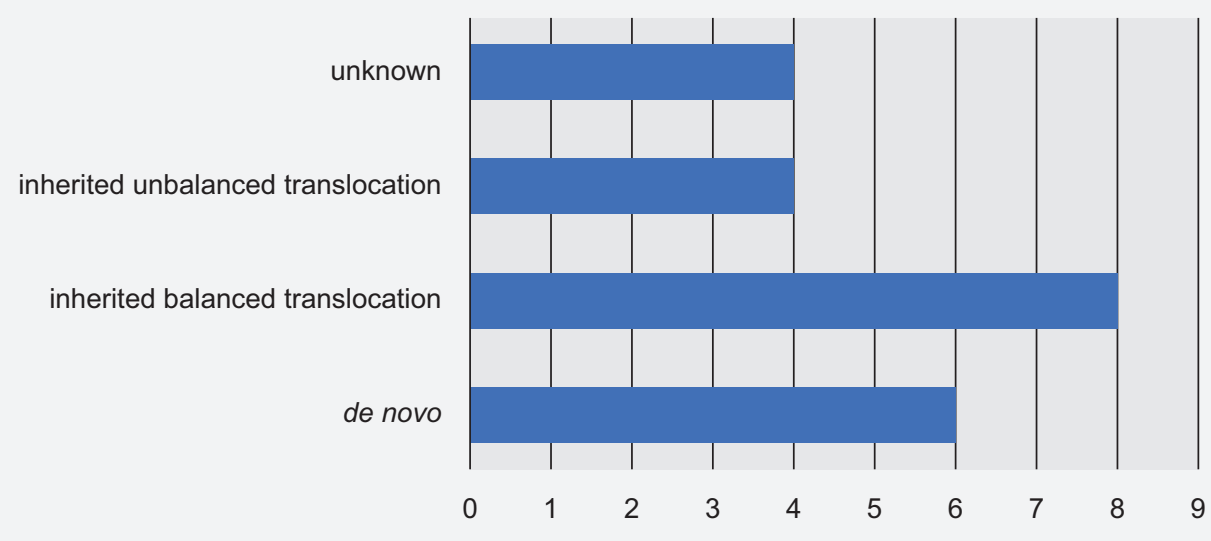

Figura 3 - Cytogenetic characteristics 

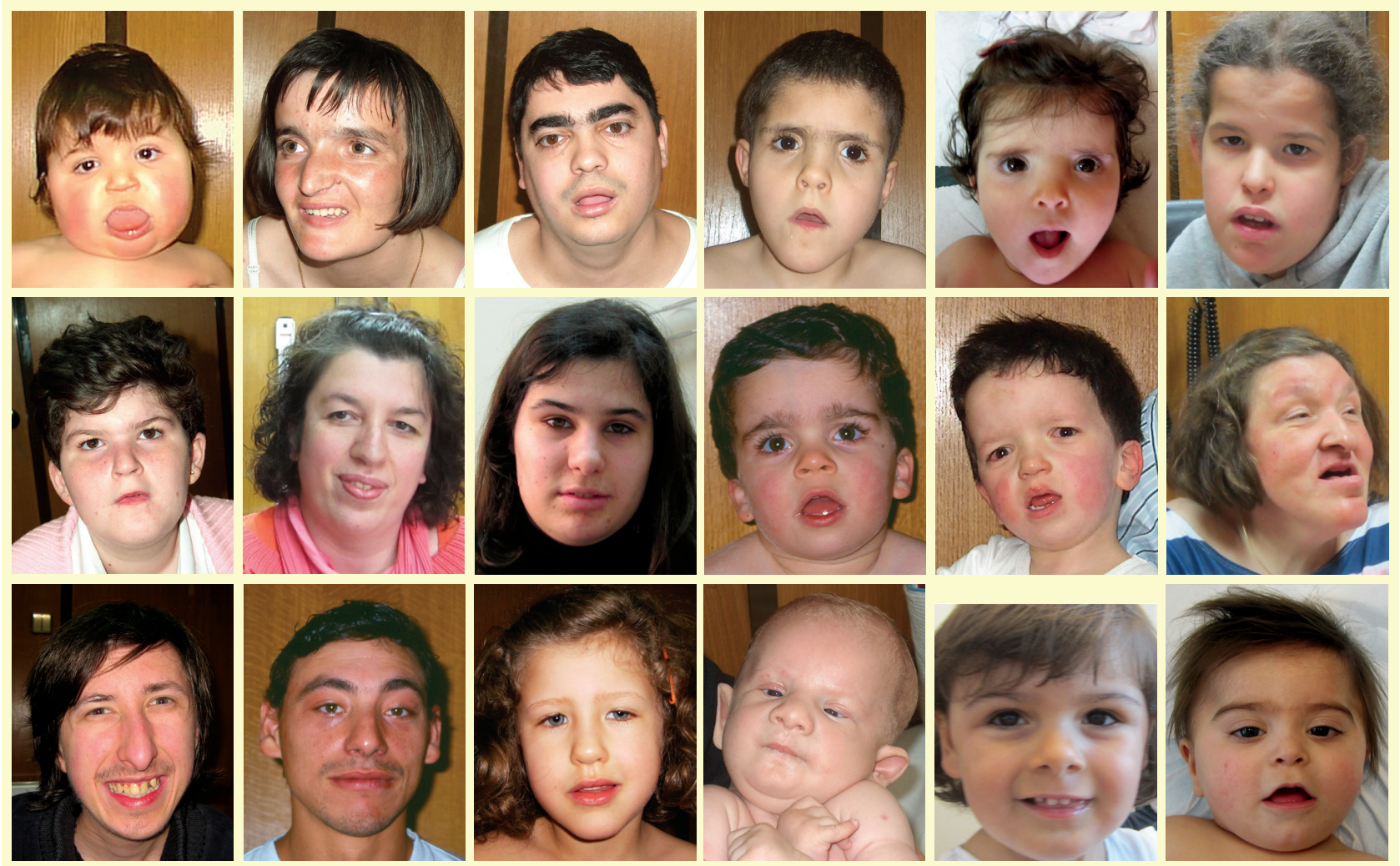

Figure 4 - Photos of patients showing dysmorphic features: cases number 21, 2, 3, 4, 5, 7, 8, 9, 10, 11, 12, 13, 14, 15, 17, 18, 19, 20. For all these cases, written informed consent was given to publish the photographs.

In fact, the translocation between the subtelomeric regions of chromosomes 4 and 8 is considered the second most common in humans. The $8 p 23$ duplication has been associated with ID, autism and psychiatric manifestations, as well as dysmorphisms (such as high forehead, epicanthic folds, hypertelorism, small eyes, strabismus, long philtrum, micrognathia, low-set malformed ears) and cardiac or renal malformations. The 4p16.3 duplication causes mild developmental delay/ID, speech delay, growth delay and mild dysmorphic features (hypertelorism, epicanthic folds and abnormal ears). Both cases have phenotypic manifestations that are very different, and it is hard to categorize their characteristics into a specific syndrome. Patient 19 seems to fit better into $4 \mathrm{p} 16.3$ duplication characteristics. ${ }^{20-22}$

Both cases 11 and 12 presented with chromosome 6pter-p24 deletion syndrome, characterized by ID, ophthalmologic abnormalities, craniofacial dysmorphisms (macrocephaly, prominent forehead, down-slanting palpebral fissures, hypertelorism and depressed nasal bridge), Dandy-Walker malformation, congenital heart defects, hypotonia, hearing loss, and others, with high phenotypic variability. ${ }^{23,24}$ The father of case 12 presented the same phenotype as his son.

Case 15 was more compatible with chromosome 15q26qter deletion syndrome, characterized by pre and postnatal growth retardation, variable ID, mild non-specific facial dysmorphisms and other congenital abnormalities. ${ }^{25}$

Case 18's manifestations seem to be caused mainly by $9 p$ duplication as patients with this syndrome present with peculiar facial dysmorphisms and digital abnormalities, as well as variable developmental delay/ID, cardiac and skeletal abnormalities. ${ }^{27}$

Case 20 could be misclassified as classic Down syndrome, since failure to thrive and other malformations (such as cardiac abnormalities) were missing. The 21 q22 duplication was the dominant phenotype. ${ }^{28}$

Case 21 was clinically diagnosed as BeckwithWiedemann syndrome. This syndrome is mainly characterized by macrosomia, macroglossia, hemihyperplasia, omphalocele, visceromegaly, embryonal tumours and neonatal hypoglicemia. ${ }^{29}$

Cases 13, 16 and 17 presented with characteristics from both deletion and duplication and it was not possible to detect predominance of either (Table 1).

Focusing on genetic screening and counselling of the family, it is important to remember the examples of cases 4 , 5 and 6 and case 21 . The first family is a good illustration of how balanced translocations may originate unbalanced gametes and affect different members of the same family. Thus, it is essential to study those at risk. The second example shows an apparently innocuous family history but in whom the translocation is present and transmitted throughout three generations.

In the aCGH era, it is tempting to perform only this technique in both children and parents to confirm a de novo occurrence; however, a normal aCGH result does not exclude a balanced translocation in parents, leaving the family at risk for chromosomal imbalances in future pregnancies of 
healthy carriers. It is essential to study parents, by performing karyotype and FISH techniques when a deletion or duplication is found in a subtelomeric region, particularly when both a deletion and duplication are found in the proband, because the probability that a balanced rearrangement is present in one of the parents is very high in this situation.

\section{CONCLUSION}

This study shows that only after complete genetic characterization is it possible to identify other relatives at risk and offer accurate genetic counselling and reproductive options, particularly invasive prenatal testing (either by chorionic villous sampling or amniocentesis) or preimplantation genetic diagnosis.

The authors wish to emphasize that, although rare, these subtelomeric changes constitute a substantial cause of syndromic ID with important familial repercussions. It is essential to be aware of these cases and refer them to a Medical Genetics consultation, so that familial studies and management can be carried out accurately.

\section{REFERENCES}

1. Miller DT, Adam MP, Aradhya S, LG Biesecker, AR Brothman, NP Carter, et al. Consensus statement: chromosomal microarray is a first-tier clinical diagnostic test for individuals with developmental disabilities or congenital anomalies. Am J Hum Genet. 2010;86,749-64.

2. Freitas MM, Silva ML, Ribeiro J, Oliva-Teles N, Candeias C, Bronzeda-Rocha $\mathrm{E}$. Análise das regiões subteloméricas em 1180 doentes com atraso mental por FISH e MLPA. Arq Med. 2013;27:10-4.

3. de Vries BB, White SM, Regan R, Homfray T, Young ID, Super M, et al. Clinical studies on submicroscopic subtelomeric rearrangements: a checklist. J Med Genet. 2001;38:145-50.

4. Manning M, Hudgins L. Use of array-based technology in the practice of medical genetics. Genet Med. 2007;9:650-3.

5. Diagnostic and Statistical Manual of Mental Disorders (DSM-5). Washington: American Psychiatric Publishing, Inc; 2013.

6. Esparza-Garrido RR, Velázquez-Wong AC, Araujo-Solís MA, Huicochea-Montiel JC, Velázquez-Flores MÁ, Salamanca-Gómez F, et al. Duplication of the Miller-Dicker critical region in a patient with a subtelomeric unbalanced translocation $t(10 ; 17)(p 15.3 ; p 13.3)$. Mol Syndromol. 2012;3:82-8.

7. Knight SJ, Flint J. Perfect endings: a review of subtelomeric probes and their use in clinical diagnosis. J Med Genet. 2000;37:401-9.

8. Manning M, Hudgins L, Professional Practice and Guidelines Committee. Array-based technology and recommendations for utilization in medical genetics practice for detection of chromosomal abnormalities. Genet Med. 2010;12:742-5.

9. Darilek S, Ward P, Pursley A, Plunkett K, Furman P, Magoulas P, et al. Pre- and postnatal genetics testing by array-comparative genomic hybridization: genetic counselling perspectives. Genet Med. 2008;10:13-8.

10. Ravnan JB, Tepperberg JH, Papenhausen P, Lamb AN, Hedrick J, Eash $\mathrm{D}$, et al. Subtelomere FISH analysis of 11688 cases: an evaluation of the frequency and pattern of subtelomere rearrangements in individuals with developmental disabilities. J Med Genet. 2006;43:478-89.

11. Digilio MG, Bernardini L, Capalbo A, Capolino R, Gagliardi MG, Marino $B$, et al. $16 p$ subtelomeric duplication: a clinically recognizable syndrome. Eur J Hum Genet. 2009;17:1135-40.

12. Phelan K, McDermid HE. The 22q13.3 deletion syndrome (PhelanMcDermid syndrome). Mol Syndromol. 2012;2:186-201.

13. Samanich J, Montagna C, Morrow BE, Babcock M. Interstitial duplication of 22q13.2 in a girl with short stature, impaired speech and language, and dysmorphism. J Pediatr Genet. 2012;1:47-53.

14. Mainardi PC, Perfumo C, Calì A, Coucourde G, Pastore G, Cavani S, et al. Clinical and molecular characterisation of 80 patients with $5 p$ deletion: genotype-phenotype correlation. J Med Genet. 2001;38:151-8.

15. Rudnik-Schoneborn S, Zerres K, HAusler M, Lott A, Krings T, Schuler $\mathrm{HM}$. A new case of proximal monosomy $1 \mathrm{p} 36$, extending the phenotype. Research letter. Am J Med Genet A. 2008;146A:2018-22.

\section{PROTECTION OF HUMANS AND ANIMALS}

The authors declare that the procedures were followed according to the regulations established by the Clinical Research and Ethics Committee and to the Helsinki Declaration of the World Medical Association.

\section{DATA CONFIDENTIALITY}

The authors declare having followed the protocols in use at their working center regarding patients' data publication. Patient consent obtained.

\section{CONFLICTS OF INTEREST}

All authors report no conflict of interest.

\section{FUNDING SOURCES}

This research received no specific grant from any funding agency in the public, commercial, or not-for-profit sectors.

16. Watanabe $M$, Hayabuchi $\mathrm{Y}$, Ono A, Naruto $\mathrm{T}$, Horikawa $\mathrm{H}$, Kohmoto $\mathrm{T}$, et al. Detection of 1p36 deletion by clinical exome-first diagnostic approach. Hum Genome Var. 2016;3:16006

17. Cho EK, Kim J, Yang A, Cho SY, Jin DK. 2q37 Deletion syndrome confirmed by high-resolution cytogenetic analysis. Ann Pediatr Endocrinol Metab. 2017;22:129-32.

18. Mehraein Y, Pfob M, Steinlein O, Aichinger E, Eggert M, Bubendorff V, et al. 2q37.3 deletion syndrome: two cases with highly distinctive facial phenotype, discordant association with schizophrenic psychosis, and shared deletion breakpoint region on 2q37.3. Cytogenet Genome Res. 2015;146:33-8.

19. Vaglio A, Milunsky A, Huang XL, Quadrelli A, Mechoso B, Quadrelli R. A fourteen years follow-up of a case of partial trisomy $12 q$ and monosomy $12 p$ recombinants of a familial pericentric inversion of chromosome 12 : clinical, cytogenetic and molecular observations. Eur J Med Genet. 2007:50:224-32.

20. Sagar A, Pinto D, Najjar F, Guter SJ, Macmillan C, Cook EH. De novo unbalanced translocation ( $4 p$ duplication/8p deletion) in a patient with autism, OCD, and overgrowth syndrome. Am J Med Genet A. 2017; 173:1656-62.

21. Skrlec I, Wagner J, Pubeljić S, Heffer M, Stipoljev F. De novo case of a partial trisomy $4 p$ and a partial monosomy 8p. Coll Antropol. 2014;38:319-23.

22. Carmany EP, Bawle EV. Microduplication of $4 p 16.3$ due to an unbalanced translocation resulting in a mild phenotype. Am J Med Genet A. 2011;155A:819-24.

23. Lin RJ, Cherry AM, Chen KC, Lyons M, Hoyme HE, Hudgins L. Terminal deletion of $6 p$ results in a recognizable phenotype. Am J Med Genet A. 2005;136:162-8.

24. Descipio C, Schneider L, Young TL, Wasserman N, Yaeger D, Lu F, et al. Subtelomeric deletions of chromosome $6 \mathrm{p}$ : molecular and cytogenetic characterization of three new cases with phenotypic overlap with Ritscher-Schinzel (3C) syndrome. Am J Med Genet A. 2005;134A:3-11.

25. Reiss R, Ahern D, Sandstrom M, Wilkins-Haug L. Recurrent enlarged nuchal translucency: First trimester presentation of a familial $15 q 26 \rightarrow$ qter deletion. Am J Med Genet A. 2014;167A:612-6.

26. Guilherme RS, Meloni VA, Perez AB, Pilla AL, Ramos MA, Dantas AG, et al. Duplication $9 p$ and their implication to phenotype. BMC Med Genet. 2014;15:142.

27. Tonnies H, Schulze I, Hennies H, Neumann LM, Keitzer R, Neitzel H. De novo terminal deletion of chromosome 15q26.1 characterised by comparative genomic hybridisation and FISH with locus specific probes. J Med Genet. 2001;38:617-21.

28. Asim A, Kumar A, Muthuswamy S, Jain S, Agarwal S. Down syndrome: an insight of the disease. J Biomed Sci. 2015;22:41.

29. Gicquel C, Rossignol S, Le Bouc Y. Beckwith-Wiedemann syndrome. Orphanet encyclopedia. 2005. 